\title{
HDAC inhibitor 4-phenylbutyrate preserves immature phenotype of human embryonic midbrain stem cells: Implications for the involvement of DNA methyltransferase
}

\author{
ZAHIDUL KHAN ${ }^{1}$, MONIRA AKHTAR and TOMAS J. EKSTRÖM \\ Department of Clinical Neuroscience, Center for Molecular Medicine, Karolinska Institutet, \\ Karolinska University Hospital, Solna, SE-17176 Stockholm, Sweden
}

Received June 15, 2011; Accepted July 18, 2011

DOI: $10.3892 / \mathrm{ijmm} .2011 .791$

\begin{abstract}
Cell replacement and gene therapy using neural stem cells (NSCs) have been widely touted as a promising treatment for CNS diseases including brain tumors. Histone deacetylase (HDAC) inhibitors have been used to explore mechanisms behind the lineage-specific differentiation of NSCs and as modulators of gene therapy. We have used the human embryonic midbrain stem cell line NGC-407 and the HDAC inhibitor 4-phenylbutyrate (4-PB) to investigate the differentiation from epigenetic perspectives. NGC-407 cells can differentiate into both neurons and glial cells, evidenced by morphological characteristics as well as up-regulation of the respective markers $\beta$-tubulin III and glial fibrillary acidic protein (GFAP) and simultaneous down-regulation of the NSC-marker nestin. Genomic DNA extracted from the differentiating cells was globally more methylated than that of the proliferating cells. The differentiating cells showed increased expression of the de novo DNA methyltransferase DNMT3B along with strong immunoreactivity in the cell nuclei. When these cells were treated with 4-PB, both the astrocytic and the neuronal differentiation phenotypes were suppressed, which paralleled a substantially weakened DNMT3B immunoreactivity in the cell nuclei. Importantly, 4-PB treatment preserves the immature phenotype of these differentiating cells as indicated by Western blot analysis and immunocytochemical analyses of the NSC markers, nestin and CD133. Nestin becomes entirely degraded 5 days after induction of differentiation, but upon exposure to 4-PB, some of the differentiating cells retain the integrity of
\end{abstract}

Correspondence to: Dr Tomas J. Ekström, Department of Clinical Neuroscience, Center for Molecular Medicine, Karolinska Institutet, Building L8:01, Karolinska University Hospital, Solna, SE-17176 Stockholm, Sweden

E-mail: tomas.ekstrom@ki.se

Present address: ${ }^{1}$ Department of Medicine, Center for Molecular Medicine, Karolinska Institutet, Building L8:03, Karolinska University Hospital, Solna, SE-17176 Stockholm, Sweden

Key words: neural stem cells, differentiation, histone deacetylase, DNA methyltransferase, 4-phenylbutyrate nestin and concurrently, CD133 is also up-regulated. Taken together, the data suggests that HDAC activity is necessary for human embryonic NSC differentiation.

\section{Introduction}

Neural stem cells (NSCs) hold great promise for the treatment of CNS diseases, including brain tumors. Exploiting their inherent biology such as multipotency and tropism, NSCs could be utilized to replace and rescue damaged and sick brain cells or to carry and deliver therapeutic molecules into brain tumors (1-5). Investigators are now focusing on exploring mechanisms behind the specificity of fate and tropism of NSCs in response to distress signals. The radically different gene programs, expression profiles, and highly specialized functions in differentiated cells compared to embryonic precursors underscore the importance of epigenetic mechanisms, including histone modifications and DNA methylation, in the differentiation processes.

Histone acetylation attained by converse activities of histone acetyltransferases (HATs) and histone deacetylases (HDACs) is associated with regulation of gene expression (6). HDAC inhibitors induce hyperacetylation and selectively modulate gene expression through altered chromatin structure making genes available for transcription (7). HDAC activity is reported to be necessary for embryonic stem cells and hematopoietic stem cell differentiation $(8,9)$. Deletion of both HDAC1 and HDAC2 completely blocks neuronal differentiation in a mouse model (10). An up-regulated HDAC activity is also found when embryonic rodent hippocampal progenitors are differentiated into neurons and astrocytes (11). Accordingly, the HDAC inhibitors trichostatin A (TSA) and valproic acid inhibit the astrocytic and oligodendrocytic differentiation of rodent NSCs (12-14) and promotes the self-renewal of hematopoietic stem cells (15).

DNA methylation is carried out by DNA methyltransferases (DNMTs). DNMT3A and DNMT3B mainly catalyze the de novo methylation and DNMT1 preferentially methylates hemi-methylated DNA to maintain existing methylation patterns after each cell division (16). DNA methylation is crucial for normal embryonic development and thus for the differentiation processes $(17,18)$. For example, demethylation 
of the promoter region of the astrocytic gene glial fibrillary acidic protein (GFAP) activates astrocytic differentiation of forebrain progenitors (19). Interestingly, the mechanisms of DNA methylation and histone acetylation are tightly coordinated (20-22). Chromatin regions which contain hypoacetylated histones also normally contain hypermethylated DNA (23). Furthermore, chemical compounds such as TSA and sodium butyrate which inhibit HDAC activity, induce DNA demethylation (24-26).

In a previous study, we presented the human embryonic midbrain progenitor cell line NGC-407 and showed that HDAC inhibitors can be used as modulators of NSC-mediated cancer gene therapy (27). Roybon et al (28) have reported the differentiation potential of NGC-407 cells by manipulating transcription factors. Considering the pivotal role of epigenetic mechanisms in the differentiation process, we studied phenotypic and global epigenetic changes in NGC-407 cells over 5 days during their differentiation, and after epigenetic manipulation with the HDAC inhibitor, 4-phenylbutyrate (4-PB).

\section{Materials and methods}

Cell culture, differentiation and drug treatment. The NGC-407 cell line was established and cultured as described elsewhere $(27,28)$. Briefly, the cells were isolated from the ventral mesencephalon of a 7-week-old human embryo (in accordance with Lund University ethics guidelines) and immortalized by the $\mathrm{v}$-myc oncogene. The cells were cultured as proliferating cells in DMEM-F12 (1:1) with Glutamax I medium (Gibco, 31331028) supplemented with $40 \mathrm{ng} / \mathrm{ml}$ basic fibroblast growth factor (bFGF) (R\&D Systems, 233-FB), $20 \mathrm{ng} / \mathrm{ml}$ epidermal growth factor (EGF) (Invitrogen, 13247-051), 1X N-2 supplement (Invitrogen, 17502-048), 0.5\% human serum albumin (Sigma, A1653), 6 g/l glucose (Sigma, G8769), $5 \mu$ M HEPES buffer solution, (Invitrogen, 15630-56) and 1X non-essential amino acids (Invitrogen, 11140-035). The cells were differentiated for 5 days by withdrawing bFGF and EGF $24 \mathrm{~h}$ after the seeding. 4-PB was purchased from Triple Crown USA and dissolved in phosphate-buffered saline (PBS). A final concentration of $0.5 \mathrm{mM}$ of 4-PB solution was used to treat the proliferating and differentiating cells.

Immunocytochemistry. Proliferating and differentiating NGC-407 cells were grown on cell culture glass slides. The cells were fixed with phosphate-buffered $4 \%$ formaldehyde for 15 min followed by washing in PBS three times. Cells were permeabilized with $0.1 \%$ Triton X-100 in PBS for 10 min and non-specific binding sites were blocked for $1 \mathrm{~h}$ with $1 \%$ bovine serum albumin in PBS supplemented with $0.1 \%$ Tween-20. The following antibodies at specified dilutions were used for primary immune-labeling: mouse monoclonal human specific anti-nestin (1:200; Abcam, ab22035), rabbit polyclonal anti-GFAP (1:500; Dako, Z0334), mouse monoclonal anti- $\beta$-tubulin III (1:500; Nordic Biosite, MMS-435P), rabbit polyclonal anti-DNMT1 (1:100; Santa Cruz Biotechnology, sc-20701), chicken polyclonal anti-DNMT3A (1:200; Chemicon, AB3431) and chicken polyclonal anti-DNMT3B (1:200; Chemicon, AB3433). Fluorochrome-conjugated secondary antibodies were as follows: $\mathrm{Cy} 3$-conjugated anti- mouse IgG (1:1,000; Amersham, PA43002), Cy3-conjugated anti-rabbit IgG (1:500; Jackson Immunoresearch Laboratories, Inc.), Cy3-conjugated anti-chicken IgG and FITC-conjugated anti-chicken IgY (1:100; Chemicon, AP194F). Hoechst 33342 $(0.05 \mu \mathrm{g} / \mathrm{ml}$; Molecular Probes, H3570) was used as nuclear counter staining. Bound antibodies were visualized by the Olympus BX50WI or Zeiss Axioplan 2 fluorescence microscopes.

Protein extraction and Western blot analysis. Proliferating and differentiating NGC-407 cell pellets were solubilized in $100 \mu 1$ lysis buffer containing $2 \%$ sodium dodecyl sulphate, $10 \%$ glycerol, $0.05 \mathrm{mM}$ PMSF and protease inhibitors (Roche, 1836153 ) in $250 \mathrm{mM}$ Tris- $\mathrm{HCl}, \mathrm{pH}$ 8.5. Protein concentration of the cell lysates was quantified using the micro BCA protein assay kit (Pierce). Aliquots of cell lysates were mixed with loading buffer containing $1 \% \beta$-mercaptoethanol, $0.02 \%$ bromophenol blue, and $0.1 \mathrm{M}$ dithiothreitol. Equal amounts of protein $(50 \mu \mathrm{g} / \mathrm{lane})$ were loaded on $6-10 \%$ polyacrylamide gels, separated by sodium dodecyl sulphate-polyacrylamide gel electrophoresis (SDS-PAGE), and electrically transferred onto nitrocellulose membranes (Schleicher and Schuell) with a transblot apparatus (Bio-Rad). Equal loading of proteins was verified by staining the membranes with Ponceau $\mathrm{S}$ solution ( $0.2 \%$ Ponceau S, 3\% trichloroacetic acid and 3\% sulphosalicylic acid), visualized by a CCD camera followed by computerized densitometry and measurement of $\beta$-actin levels. After washing away the Ponceau stain, the membranes were blocked for $30 \mathrm{~min}$ with $5 \%$ non-fat dry milk dissolved in Tris-HCl-buffered saline supplemented with $0.05 \%$ Tween-20 (TBST). Immune-labeling was performed with the following primary antibodies at specified dilutions: mouse monoclonal human specific anti-nestin (1:1,000; Abcam, ab22035), mouse monoclonal anti-human CD133 (1:200; Miltenyi Biotec), mouse monoclonal anti-GFAP (1:1,000; BD Pharmingen, 556327), mouse monoclonal anti- $\beta$-tubulin III (1:1,000; Nordic Biosite, MMS-435P), rabbit polyclonal anti-DNMT1 (1:1,000; Santa Cruz Biotechnology, sc-20701), chicken polyclonal antiDNMT3A (1:1,000; Chemicon, AB3431), chicken polyclonal anti-DNMT3B (1:1,000; Chemicon, AB3433) and mouse monoclonal anti- $\beta$-actin (1:3,000, Novus Biologicals, NB600501). Following three TBST washings, the membranes were incubated with secondary antibodies coupled to horseradish peroxidase (HRP) which were as follows: anti-mouse IgG-HRP (1:3,000, Bio-Rad), anti-rabbit IgG-HRP (1:3,000, Bio-Rad), anti-chicken IgY-HRP (1:2,000; Abcam, ab6877). Bound antibodies were detected by an enhanced chemiluminescence detection kit (Amersham) and visualized by a CCD camera using the IR LAS-1000 Pro software (Ver. 2.2, FujiFilm). Acquired images were analyzed with the Image Gauge (Ver. 3.12 , FujiFilm) to quantify the signals. Statistical significance between means was assessed by the Student's t-test for unpaired values.

Measurement of global DNA methylation by a luminometric methylation assay (LUMA). Genomic DNA of proliferating and differentiating NGC-407 cells cultured with or without 4-PB was extracted using the GeneElute Mammalian Genomic DNA Miniprep kit (Sigma). DNA was quantified using the NanoDrop ND-1000 spectrophotometer. Global DNA methylation was 
A
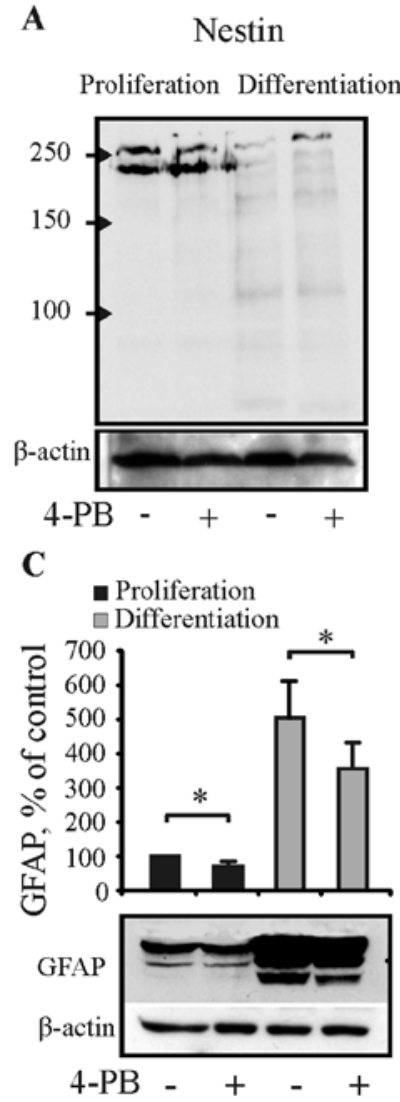

\section{B}

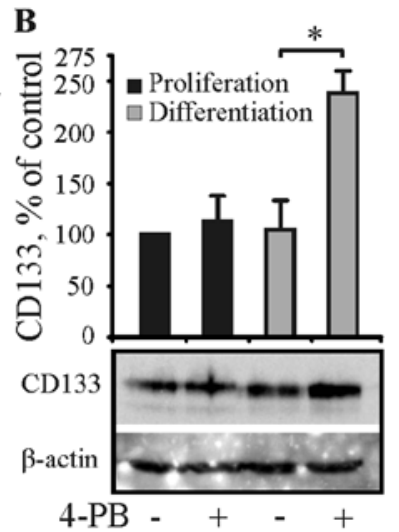

D

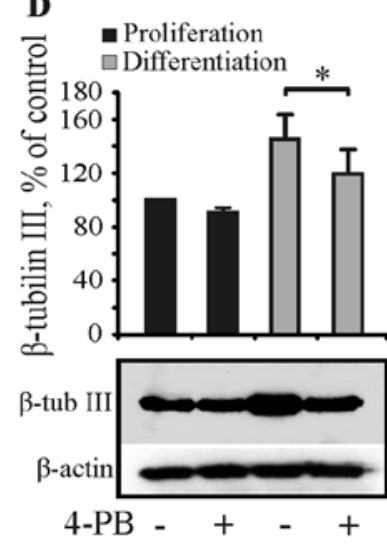

Figure 1. Effects of 4-PB on neural stem cell and differentiated cell markers in NGC-407 cells analyzed by Western blot analysis. NGC-407 cells were exposed to $0.5 \mathrm{mM}$ of 4 -PB for 5 days while growing as proliferating cells or being differentiated by removing growth factors from the culture media. Western blot analyses show (A and B) the neural stem cell marker nestin and CD133 respectively, $(C)$ the astrocyte marker glial fibrillary acidic protein (GFAP) and (D) the neuronal marker $\beta$-tubulin III. $\beta$-actin levels demonstrate the accuracy of protein loading. Bar graphs represent the means and error bars denote \pm SEM from three independent experiments. Statistical significance between means was assessed by the Student's t-test for unpaired values. Comparisons were made between 4-PB treated and untreated NGC-407 cells. Note that in the differentiating cells, nestin becomes almost entirely degraded, but upon exposure to 4-PB, some of its longer form remains intact. 4-PB also up-regulates CD133 in these cells while GFAP and $\beta$-tubulin III become down-regulated. Equal gel loading for all Western blot analyses, was verified by Ponceau staining and computerized densitometry. ${ }^{*} \mathrm{P}<0.05$.

assessed by LUMA as described in detail elsewhere $(29,30)$. Briefly, genomic DNA (200-500 ng) was separately cleaved with HpaII $+E c o$ RI and $M s p \mathrm{I}+E c o \mathrm{RI}$ and the degree of cleavage was subsequently quantified on the Pyrosequencing ${ }^{\mathrm{TM}}$ platform using PSQ96 ${ }^{\mathrm{TM}}$ MA system and software (Biotage $\mathrm{AB}$ ). The calculated ratio of $\mathrm{HpaII} / \mathrm{MspI}$ (ranging from 0 to 1 ) represents the degree of global DNA methylation; the greater the HpaII/MspI ratio, the lower the degree of methylation, and vice versa.

\section{Results}

Effects of 4-phenylbutyrate on neural stem cell markers. Mitogen withdrawal from culture media leads to differentiation of NGC-407 cells (27). We treated NGC-407 cells with 4-PB in the presence or absence of EGF and bFGF to investigate the 4-PB effect in both proliferating and differentiating cells,

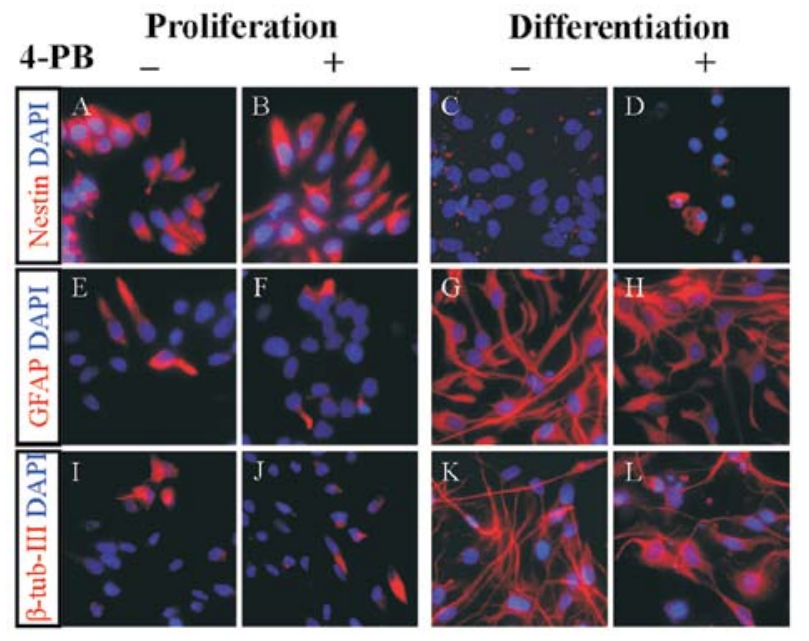

Figure 2. 4-PB inhibits differentiation and preserves the immature phenotype of NGC-407 cells. NGC-407 cells were grown as proliferating cells or were induced to differentiate for 5 days, and treated with $0.5 \mathrm{mM}$ of $4-\mathrm{PB}$ The neural stem cell marker nestin, the astrocyte marker glial fibrillary acidic protein (GFAP) and the neuronal marker $\beta$-tubulin III ( $\beta$-tub-III) were analyzed by immunocytochemistry. Images (A-D) show nestin (red); (E-H) GFAP (red) and (I-L) $\beta$-tub-III (red). In all images, DAPI (blue) shows the nuclear counterstaining. $(\mathrm{G}$ and $\mathrm{H})$ and $(\mathrm{K}$ and $\mathrm{L})$ show cluster of cells expressing GFAP and $\beta$-tub-III respectively. (D) Note that after 4-PB treatment, some differentiating cells preserve the integrity of nestin. Growth of astrocytic and neuronal processes is also suppressed in some of the differentiating cells upon exposure to 4-PB ( $\mathrm{H}$ and L). Magnification, x60.

respectively. Western blot analysis of the whole-cell extracts of NGC-407 cells detected two immunoreactive forms of the neural stem cell marker nestin using a human-specific nestin antibody. The short form (faster migration on SDS-PAGE), presumably the unmodified nestin, was predominant in the proliferating NGC-407 cells (Fig. 1A). Induction of differentiation led to apparent down-regulation and degradation of nestin within 5 days. Interestingly, when these differentiating cells were treated by 4-PB, the longer form of nestin remained intact, although the short, unmodified form, was diminished (Fig. 1A). Significant up-regulation of another neural stem cell marker, CD133, was also observed in the differentiating cells upon exposure to 4-PB (Fig. 1B). Immunocytochemical analysis showed that some NGC-407 cells in the differentiating culture preserved the integrity of substantial nestin expression when treated with 4-PB, although it caused no substantial change in nestin reactivity in the proliferating cells (Fig. 2A-D).

Effects of 4-phenylbutyrate on astrocytic and neuronal differentiations of $\mathrm{NGC}-407 \mathrm{cells}$. We then investigated the astrocytic marker GFAP and the neuronal marker $\beta$-tubulin III, by both Western blot analysis and immunocytochemical analyses, to examine the 4-PB effect during the progression of differentiation of NGC-407 cells. A few proliferating NGC-407 cells expressed both of these markers, although they showed an immature phenotype of possessing short cell processes and no network formation (Figs. 1C and D and 2E and I). As expected, upon removal of mitogens, the NGC-407 cells differentiated to astrocytes and neurons with substantial up-regulation of the respective markers GFAP and $\beta$-tubulin III (Fig. 1C and D) and formation of extended cell processes and delicate networks 


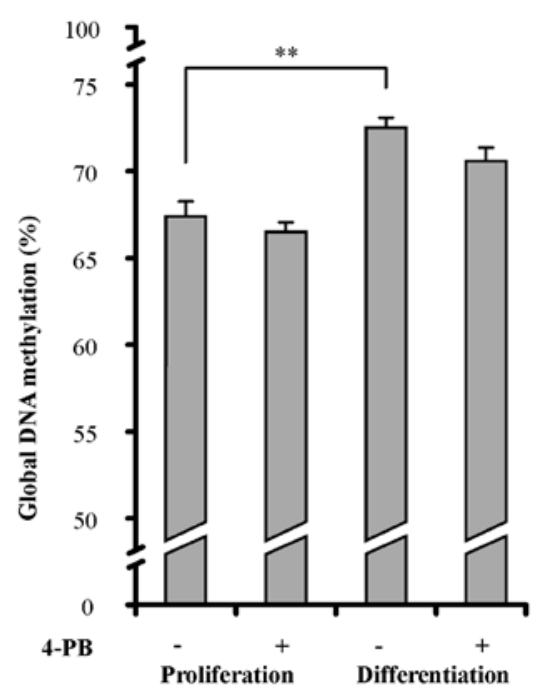

Figure 3. Global DNA methylation level of NGC-407 cells upon differentiation and exposure to 4-PB. Genomic DNA from proliferating and differentiating NGC-407 cells cultured with or without $0.5 \mathrm{mM}$ 4-PB, was analyzed by LUMA. Bar graphs represent mean global DNA methylation levels calculated from $H p a I I / M s p I$ ratios and error bars denote the SEM from three independent experiments. ${ }^{* * *} \mathrm{P}<0.01$.

(Fig. 2G and K). We found that when the cells were treated with 4-PB, GFAP was significantly down-regulated in both proliferating and differentiating cells (Fig. $1 \mathrm{C})(\mathrm{P}<0.05)$. The $\beta$-tubulin III expression was significantly down-regulated in the differentiating cells $(\mathrm{P}<0.05)$ indicating that $4-\mathrm{PB}$ prevents neuronal differentiation (Fig. 1D). Immunostaining with GFAP and $\beta$-tubulin III suggested that 4-PB suppressed the progression of both astrocytic (Fig. 2G and H) and neuronal (Fig. 2K and $\mathrm{L}$ ) differentiation, as indicated by morphological characteristics, such as arrested development of cell processes and absence of a fine network formation.

Global DNA methylation level in NGC-407 cells. LUMA analysis revealed that the differentiating NGC-407 cell genome was significantly more methylated compared to that of the proliferating cells $(\mathrm{P}<0.01)$ (Fig. 3). When the cells were treated with 4-PB, a slight trend of decrease in the global DNA methylation level was observed in both the differentiating and the proliferating cells, compared with the corresponding untreated cells (Fig. 3).

Effects of 4-phenylbutyrate on DNA methyltransferases. Western blot analysis of whole-cell extracts of NGC-407 cells detected two isoforms of DNMT1 under proliferating conditions. The longer isoform of DNMT1 is predominant in the proliferating NGC-407 cells. However, upon differentiation for 5 days, this longer form disappeared completely and the short form became up-regulated. When the cells were treated with 4-PB, the predominant longer form of DNMT1 was substantially down-regulated in the proliferating cells while the short form remained unchanged. In the differentiating cells, a down-regulation of the existing short form of DNMT1 was observed upon exposure to 4-PB (Fig. 4). Immunocytochemical analysis showed a particulate nuclear staining of DNMT1 which was abundant in the proliferating NGC-407 cells and relatively sparse in the differentiating cells

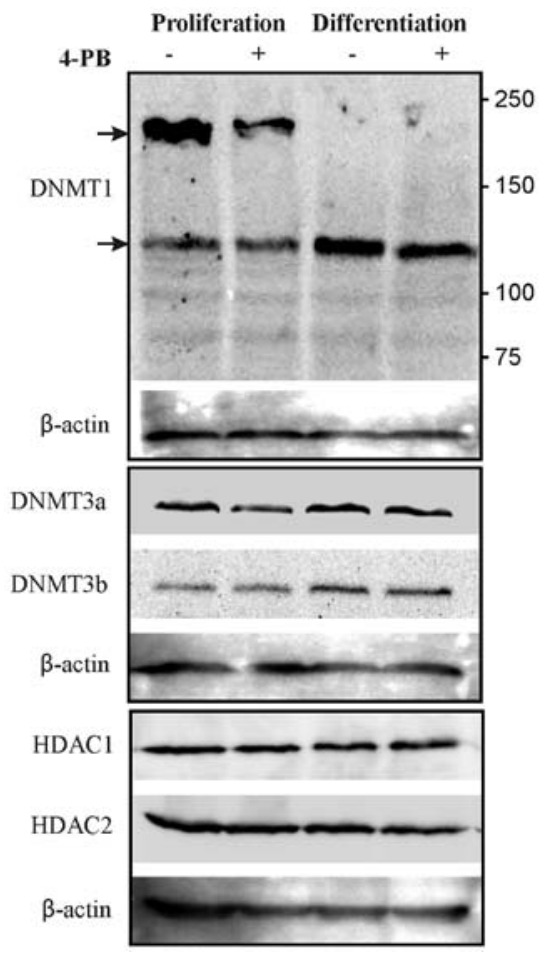

Figure 4. Western blot analysis showing the level of DNMTs in NGC-407 cells under proliferating condition and after differentiation for 5 days. The cells were also treated with $0.5 \mathrm{mM}$ of 4-PB for 5 days in both culture conditions. Note that DNMT1 is present as two isoforms in the proliferating cells and after differentiation the longer form disappears. In the differentiating cells, DNMT3B is up-regulated. Note also that 4-PB down-regulates DNMT1 in both type of cells and DNMT3A only in proliferating cells. $\beta$-actin levels show the accuracy of protein loading.

as seen in Fig. 5C and I. When the cells were treated with 4-PB, DNMT1 immunoreactivity varied among the proliferating cells (Fig. 5F); some displayed a very weak staining, and some a strong staining but with clumped and patchy appearance, and interestingly, some cells showed staining outside of the nucleus. 4-PB did not substantially affect DNMT1 immunoreactivity in the differentiating NGC-407 cells (Fig. 5L).

Upon differentiation for 5 days, there was no substantial change in the DNMT3A protein level compared to proliferative NGC-407 cells as analyzed by Western blotting. However, the DNMT3B level was increased in the differentiating cells compared with the proliferating ones. 4-PB treatment visibly down-regulated DNMT3A in the proliferating cells, but had no detectable effect on the DNMT3B level in either of the culture conditions (Fig. 4). Immunocytochemical analysis showed that DNMT3A was predominantly located at the perinuclear region and in the cytoplasm, whereas, DNMT3B was predominantly found in the nuclei of NGC-407 cells (Fig. 6). After 4-PB treatment both DNMT3A and DNMT3B immunoreactivity varied among the proliferating cells. In addition, the intensity of DNMT3B staining was substantially weakened in the nuclei of 4-PB treated proliferating and differentiating cells, compared with the corresponding untreated cells (Fig. 6).

\section{Discussion}

4-Phenylbutyrate preserves neural stem cell-phenotype. The cytoskeletal protein nestin and the cell membrane glycopro- 


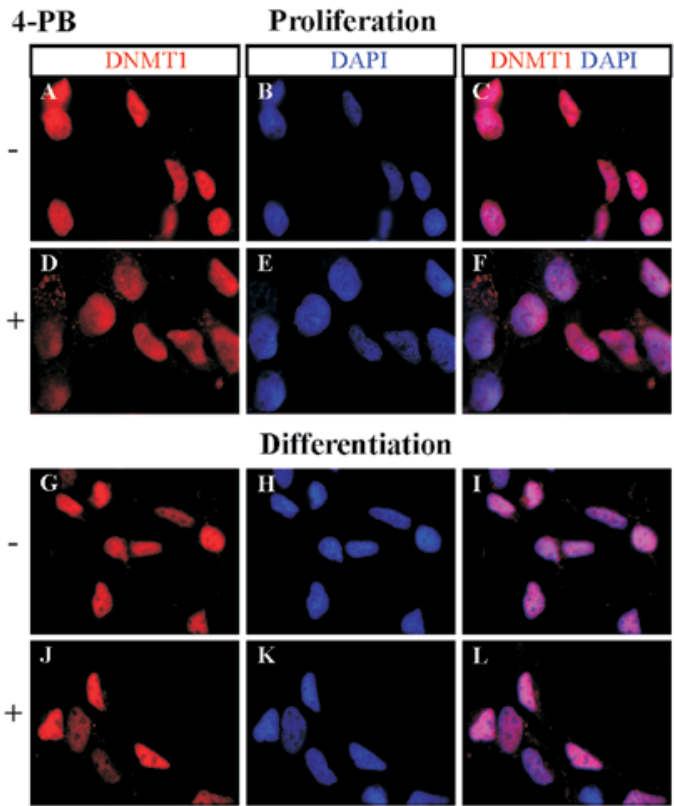

Figure 5. Immunocytochemical analysis of DNMT1 in NGC-407 cells. The cells were cultured under proliferating and differentiating conditions for 5 days with or without $0.5 \mathrm{mM}$ of 4-PB treatment. Images (A-F) are from proliferating cells and (G-L) from differentiating cells. Left panel shows DNMT1 staining in red; middle panel, nuclear counter staining DAPI in blue; and right panel, overlay images. Note the highly expressed DNMT1 in (C) the proliferating cells and $(\mathrm{F})$ greatly varied immunoreactivity among these cells upon exposure to 4-PB along with some staining outside the nuclei. Magnification, x60

tein CD133 are the most accredited markers for NSCs $(31,32)$. NGC-407 cells expressed both of these markers abundantly. Nestin was detected as two immunoreactive forms in proliferating NGC-407 cells in accordance with a previous report showing doublet nestin bands in human NSCs (33). We found that nestin in the proliferating cells became degraded within 5 days after induction of differentiation (Fig. 1), which is consistent with previous reports showing a down-regulation and subsequent elimination of nestin when embryonic NSCs exit the cell cycle and start to differentiate (34). Accordingly, nestin is considered as a marker for proliferating embryonic NSCs. Upon exposure to the HDAC inhibitor 4-PB, the integrity of nestin was retained in some of the differentiating NGC-407 cells (Figs. 1A and 2D), indicating a role for 4-PB in preserving the immature phenotype of NGC-407 cells. This corresponds with the recent report showing enhanced proliferation of NSCs after deletion of HDAC1 and HDAC2 (10). 4-PB also substantially up-regulated CD133 in the differentiating NGC-407 cells (Fig. 1B). This is interesting since a positive correlation between CD133 expression and proliferation of neuronally committed embryonic stem cells has also been reported previously (32).

4-Phenylbutyrate inhibits astrocytic and neuronal differentiation of embryonic midbrain stem cells. Consistent with the result of NSC markers, we found that 4-PB abrogated both astrocytic and neuronal differentiation of NGC-407 cells which was indicated by suppression of mature cell morphology such as a fine network of extended processes (Fig. 2). The respective markers GFAP and $\beta$-tubulin III, were also found

\section{4-PB Proliferation}

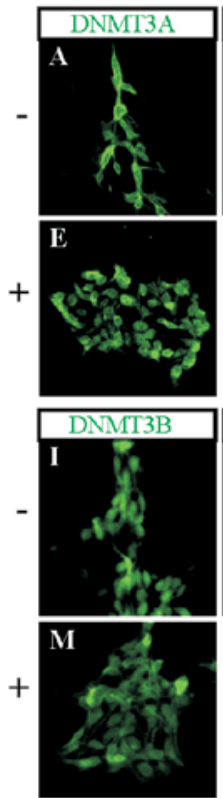

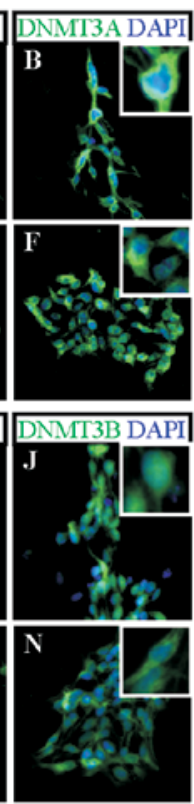

Differentiation

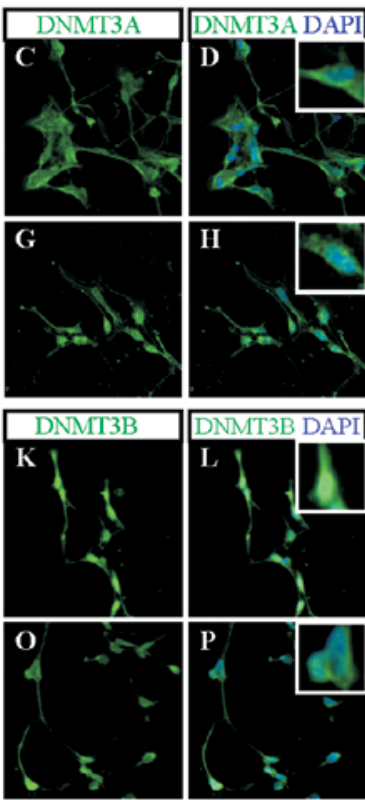

Figure 6. Immunocytochemical analysis of DNMT3A and DNMT3B in NGC-407 cells. Proliferating and differentiating cells were treated with $0.5 \mathrm{mM}$ of 4 -PB for 5 days. Images $(\mathrm{A}-\mathrm{H})$ show DNMT3A in green and nuclear counterstaining DAPI in blue. DNMT3A is predominantly expressed in the perinuclear region and in the cytoplasm in both proliferating and differentiating cells. Images (I-P) show DNMT3B in green and nuclear counterstaining DAPI in blue. DNMT3B is predominantly expressed in the nuclei of both cell phenotypes. However, note the strong immunoreactivity in the differentiating cells compared to the proliferating cells and the relocalization into the cytoplasm after 4-PB treatment. Magnification, x20 for main pictures and $3 \times 20$ for the insets.

significantly down-regulated by 4-PB treatment (Fig. 1). These findings correspond with the reports showing an important role of HDAC activity during the differentiation process of embryonic stem cells (8), which has also been demonstrated in hippocampal progenitor cells by an up-regulation of HDAC activity in differentiated neurons and astrocytes (11). Montgomery et al (10) have shown that deletion of HDAC1 and HDAC2 in a mouse model resulted in a complete inhibition of the progression of differentiation of NSCs to mature neurons. 4-PB is a non-specific HDAC inhibitor, which acts on both class I HDACs such as HDAC1 and HDAC2, as well as class II HDACs. Thus, our findings are in agreement with the report by Montgomery et al (10). TSA is another class I and II HDAC inhibitor, which is also shown to suppress NSC-differentiation into astrocytes and oligodendrocytes $(13,14)$. In contrast, the HDAC inhibitor valproic acid was shown to promote neuronal differentiation of rodent adult hippocampal progenitor cells, although a simultaneous inhibition of astrocytic differentiation indicated by decreased GFAP expression was found (12). This discrepancy of contrasting effects on NSC-differentiation by HDAC inhibitors has previously been suggested as being cell-type specific $(18,35)$. In this study we used immortalized cells derived from human embryonic midbrain stem cells, in contrast to the rodent adult hippocampal progenitor cells where a different effect of HDAC inhibitors on neuronal differentiation was reported. Additionally, adult NSCs are mainly GFAP-positive (36), depletion of which results in termination of adult neurogenesis (37). Therefore, inhibition of astrocytic 
differentiation indicated by reduced GFAP expression and concurrent stimulation of neuronal differentiation in adult NSCs is unclear.

Neural stem cell DNA becomes globally methylated upon differentiation. By the LUMA method, we measured the global DNA methylation level in the proliferating and differentiating NGC-407 cells. We found that DNA in the differentiating cells is significantly more methylated compared with the proliferating cells (Fig. 3). This is consistent with the finding that human adult brain tissue DNA is highly methylated (38). The hypermethylation upon differentiation is logically concordant with the up-regulation of the de novo DNA methyltransferase DNMT3B, and its strong immunoreactivity in the nucleus of all differentiating NGC-407 cells compared with the proliferating cells (Figs. 4 and 6K and L). During neuronal differentiation in vitro, murine Dnmt3b is known to play an upper hand role compared to the other de novo methyltransferase Dnmt3a (39). Dnmt3b knock-out mice die early in embryonic development, whereas Dnmt3a knock-out causes lethality after birth, at 4 weeks of age (40). We found two isoforms of the maintenance DNA methyltransferase DNMT1 in the proliferating NGC-407 cells. The DNMT1 antibody form used in our analysis (Santa Cruz Biotechnology, sc-20701), also identifies a similar double-band DNMT1 protein, and existence of two DNMT1 isoforms has been reported previously (41). In the differentiating cells, DNMT1 was present only as the short isoform and became up-regulated compared to the corresponding form in the proliferating cells (Fig. 4). Although DNMT1 may play a less important role in the cells, which exit the cell cycle, it has been reported that the short form of DNMT1 has higher catalytic activity than the longer form (41). This is consistent with our findings that this protein is up-regulated in differentiating cells.

Epigenetic mechanisms including histone acetylation and DNA methylation are considered as major players during cell differentiation and importantly they are found to interact with one another $(17,20)$. There are several studies showing that the HDAC inhibitors TSA and sodium butyrate induce DNA demethylation (24-26). It has also been reported that global DNA hypomethylation inhibits embryonic stem cell differentiation (42). Upon exposure to 4-PB, we found a slight loss in global DNA methylation particularly in differentiating NGC-407 cells (Fig. 3). Although the effect is small, we consider this 4-PB-induced hypomethylation as a potentially important observation in a 5 days heterogeneous cell culture system and with a low concentration of 4-PB $(0.5 \mathrm{mM})$, especially since the stem cells are known to display an unstable methylation pattern (43). It should also be noted that the LUMA assay interrogates the global methylome, and if investigating specific loci involved in the differentiation processes with e.g. bisulphite sequencing, the effects would likely be more pronounced. In addition, it has been shown that nearly $25 \%$ of the methylome in embryonic stem cells display non-CpG methylation (44) including $\mathrm{CpA}, \mathrm{CpC}$ and $\mathrm{CpT}$. Whether this is also true for the proliferating NGC-407 cells is unknown, but would certainly complicate the interpretation by LUMA which assesses $\mathrm{CpG}$ methylation in CCGG sites.

We found 4-PB-related changes in DNA methylating enzymes. In the proliferating cells, DNMT1 and DNMT3A were clearly down-regulated upon exposure to 4-PB (Fig. 4). Some of these cells showed a relatively weak immunoreactivity for DNMT1 and importantly, some DNMT1 was found to be relocated to the cytoplasm (Fig. 5). In the differentiating cells, DNMT3B which plays a pivotal role during differentiation (39), was found to relocate to the cytoplasm after 4-PB treatment (compare Fig. 6P with L), although the protein level was not changed (Fig. 4). This exit from the nucleus is a plausible explanation to the observed reduced methylation level. It remains to be investigated however, whether the hypomethylating effect of 4-PB is due to active demethylation, as has been demonstrated in cancer cell lines (26) or to the changes in expression and distribution of the methyltransferases.

In conclusion, the human embryonic midbrain progenitor cells NGC-407 become globally DNA-hypermethylated during the progression of differentiation. The HDAC inhibitor 4-PB inhibits both astrocytic and neuronal differentiation of these cells and preserves their immature phenotype, possibly involving DNMTs. Our findings also support previous reports that HDAC activity is essential for the NSC-differentiation process. If the NGC-407 cells re-differentiate after removal of 4-PB remains to be investigated. Understanding the process of NSC differentiation through epigenetic manipulation is of immense importance for meeting the expectations of NSC-mediated cell and gene therapy.

\section{Acknowledgements}

This study was supported by the Swedish Cancer Society, the Robert Lundberg's Memorial Foundation, and funds from the Karolinska Institutet. The NGC-407 cells were originally provided by Olle Lindvall and Bengt Juliusson, Lund University. We greatly acknowledge the assistance of Mohsen Karimi when running LUMA.

\section{References}

1. Blesch A and Tuszynski MH: GDNF gene delivery to injured adult CNS motor neurons promotes axonal growth, expression of the trophic neuropeptide CGRP, and cellular protection. J Comp Neurol 436: 399-410, 2001.

2. Ourednik J, Ourednik V, Lynch WP, Schachner M and Snyder EY: Neural stem cells display an inherent mechanism for rescuing dysfunctional neurons. Nat Biotechnol 20: 1103-1110, 2002.

3. Muller FJ, Snyder EY and Loring JF: Gene therapy: can neural stem cells deliver? Nat Rev Neurosci 7: 75-84, 2006.

4. Aboody KS, Brown A, Rainov NG, et al: Neural stem cells display extensive tropism for pathology in adult brain: evidence from intracranial gliomas. Proc Natl Acad Sci USA 97: 12846-12851, 2000.

5. Khan Z, Knecht W, Willer M, et al: Plant thymidine kinase 1 is an efficient suicide gene for brain tumor therapy. Neuro Oncol 12: 549-558, 2010.

6. Jenuwein T and Allis CD: Translating the histone code. Science 293: 1074-1080, 2001.

7. Johnstone RW: Histone-deacetylase inhibitors: novel drugs for the treatment of cancer. Nat Rev Drug Discov 1: 287-299, 2002.

8. Lee JH, Hart SR and Skalnik DG: Histone deacetylase activity is required for embryonic stem cell differentiation. Genesis 38: 32-38, 2004.

9. Koipally J, Renold A, Kim J and Georgopoulos K: Repression by Ikaros and Aiolos is mediated through histone deacetylase complexes. EMBO J 18: 3090-3100, 1999.

10. Montgomery RL, Hsieh J, Barbosa AC, Richardson JA and Olson EN: Histone deacetylases 1 and 2 control the progression of neural precursors to neurons during brain development. Proc Natl Acad Sci USA 106: 7876-7881, 2009. 
11. Ajamian F, Suuronen T, Salminen A and Reeben M: Up-regulation of class II histone deacetylases mRNA during neural differentiation of cultured rat hippocampal progenitor cells. Neurosci Lett 346: 57-60, 2003.

12. Hsieh J, Nakashima K, Kuwabara T, Mejia E and Gage FH: Histone deacetylase inhibition-mediated neuronal differentiation of multipotent adult neural progenitor cells. Proc Natl Acad Sci USA 101: 16659-16664, 2004.

13. Marin-Husstege M, Muggironi M,Liu A and Casaccia-Bonnefil P Histone deacetylase activity is necessary for oligodendrocyte lineage progression. J Neurosci 22: 10333-10345, 2002.

14. Shen S, Li J and Casaccia-Bonnefil P: Histone modifications affect timing of oligodendrocyte progenitor differentiation in the developing rat brain. J Cell Biol 169: 577-589, 2005.

15. Young JC, Wu S, Hansteen G, et al: Inhibitors of histone deacetylases promote hematopoietic stem cell self-renewal. Cytotherapy 6: 328-336, 2004.

16. Chen T and Li E: Structure and function of eukaryotic DNA methyltransferases. Curr Top Dev Biol 60: 55-89, 2004.

17. Li E: Chromatin modification and epigenetic reprogramming in mammalian development. Nat Rev Genet 3: 662-673, 2002.

18. Roloff TC and Nuber UA: Chromatin, epigenetics and stem cells Eur J Cell Biol 84: 123-135, 2005.

19. Takizawa T, Nakashima K, Namihira M, et al: DNA methylation is a critical cell-intrinsic determinant of astrocyte differentiation in the fetal brain. Dev Cell 1: 749-758, 2001.

20. Ng HH and Bird A: DNA methylation and chromatin modification. Curr Opin Genet Dev 9: 158-163, 1999.

21. Fuks F, Burgers WA, Brehm A, Hughes-Davies L and Kouzarides T: DNA methyltransferase Dnmt1 associates with histone deacetylase activity. Nat Genet 24: 88-91, 2000.

22. Bachman KE, Rountree MR and Baylin SB: Dnmt3a and Dnmt3b are transcriptional repressors that exhibit unique localization properties to heterochromatin. J Biol Chem 276: 32282-32287, 2001.

23. Jones PL and Wolffe AP: Relationships between chromatin organization and DNA methylation in determining gene expression. Semin Cancer Biol 9: 339-347, 1999.

24. Chen ZJ and Pikaard CS: Epigenetic silencing of RNA polymerase I transcription: a role for DNA methylation and histone modification in nucleolar dominance. Genes Dev 11: 2124-2136, 1997.

25. Selker EU: Trichostatin A causes selective loss of DNA methylation in Neurospora. Proc Natl Acad Sci USA 95: 9430-9435, 1998

26. Ou JN, Torrisani J, Unterberger A, et al: Histone deacetylase inhibitor Trichostatin A induces global and gene-specific DNA demethylation in human cancer cell lines. Biochem Pharmacol 73: 1297-1307, 2007.

27. Khan Z, Akhtar M, Asklund T, Juliusson B, Almqvist PM and Ekstrom TJ: HDAC inhibition amplifies gap junction communication in neural progenitors: potential for cell-mediated enzyme prodrug therapy. Exp Cell Res 313: 2958-2967, 2007.

28. Roybon L, Hjalt T, Christophersen NS, Li JY and Brundin P: Effects on differentiation of embryonic ventral midbrain progenitors by Lmx1a, Msx1, Ngn2, and Pitx3. J Neurosci 28: 3644-3656, 2008 .
29. Karimi M, Johansson S and Ekstrom TJ: Using LUMA: a luminometric-based assay for global DNA-methylation. Epigenetics 1: 45-48, 2006.

30. Karimi M, Johansson S, Stach D, et al: LUMA (LUminometric Methylation Assay) - a high throughput method to the analysis of genomic DNA methylation. Exp Cell Res 312: 1989-1995, 2006.

31. Gilyarov AV: Nestin in central nervous system cells. Neurosci Behav Physiol 38: 165-169, 2008.

32. Kania G, Corbeil D, Fuchs J, et al: Somatic stem cell marker prominin-1/CD133 is expressed in embryonic stem cell-derived progenitors. Stem Cells 23: 791-804, 2005.

33. Messam CA, Hou J and Major EO: Coexpression of nestin in neural and glial cells in the developing human CNS defined by a human-specific anti-nestin antibody. Exp Neurol 161: 585-596, 2000 .

34. Tohyama T, Lee VM, Rorke LB, Marvin M, McKay RD and Trojanowski JQ: Nestin expression in embryonic human neuroepithelium and in human neuroepithelial tumor cells. Lab Invest 66: 303-313, 1992.

35. Meshorer E: Chromatin in embryonic stem cell neuronal differentiation. Histol Histopathol 22: 311-319, 2007.

36. Imura T, Kornblum HI and Sofroniew MV: The predominant neural stem cell isolated from postnatal and adult forebrain but not early embryonic forebrain expresses GFAP. J Neurosci 23 : 2824-2832, 2003.

37. Garcia AD, Doan NB, Imura T, Bush TG and Sofroniew MV: GFAP-expressing progenitors are the principal source of constitutive neurogenesis in adult mouse forebrain. Nat Neurosci 7: $1233-1241,2004$

38. Ehrlich M, Gama-Sosa MA, Huang LH, et al: Amount and distribution of 5-methylcytosine in human DNA from different types of tissues of cells. Nucleic Acids Res 10: 2709-2721, 1982.

39. Bai S, Ghoshal K, Datta J, Majumder S, Yoon SO and Jacob ST: DNA methyltransferase $3 \mathrm{~b}$ regulates nerve growth factor-induced differentiation of PC12 cells by recruiting histone deacetylase 2 . Mol Cell Biol 25: 751-766, 2005.

40. Okano M, Bell DW, Haber DA and Li E: DNA methyltransferases Dnmt3a and Dnmt3b are essential for de novo methylation and mammalian development. Cell 99: 247-257, 1999.

41. Di Giaimo R, Locascio A, Aniello F, et al: DNA (cytosine-5) methyltransferase turnover and cellular localization in developing Paracentrotus lividus sea urchin embryo. Gene 272: 199-208, 2001.

42. Jackson M, Krassowska A, Gilbert N, et al: Severe global DNA hypomethylation blocks differentiation and induces histone hyperacetylation in embryonic stem cells. Mol Cell Biol 24: 8862-8871, 2004.

43. Humpherys D, Eggan K, Akutsu H, et al: Epigenetic instability in ES cells and cloned mice. Science 293: 95-97, 2001.

44. Lister R, Pelizzola M, Dowen RH, et al: Human DNA methylomes at base resolution show widespread epigenomic differences. Nature 462: 315-322, 2009. 